\title{
Fatal Tumor Lysis Syndrome Following Sorafenib Treatment
}

\author{
George Habiba, c, Munir Nashashibi ${ }^{b}$
}

\begin{abstract}
Sorafenib is one of the few approved treatments for advanced hepatocellar carcinoma. It is an oral multi-targeted tyrosine kinase inhibitor. It could be associated with a wide variety of adverse effects. However tumor lysis syndrome was reported in 2 cases only following sorafenib treatment. Here we report a fatal case of tumor lysis syndrome 3 weeks following sorafenib treatment in a patient with advanced hepatitis B virus-related hepatocellular carcinoma. We suggest using preventing measures for TLS prior to initiation of such treatment.
\end{abstract}

Keywords: Sorafenib; Tumor lysis syndrome; Hepatocellular carcinoma

\section{Introduction}

Sorafenib is an oral multi-targeted tyrosine kinase inhibitor [1]. It had been approved by the FDA for the treatment of hepatocellular carcinoma (HCC) nearly 5 years ago. It is the first approved molecular targeted agent for the treatment of HCC. Sorafenib increases survival of patients with HCC by about 2 - 3 months on average [2]. However there is a wide range of reported adverse effects following treatment with sorafenib, mostly nausea and vomiting in nearly $50 \%$ of the patients [2]. Severe adverse effects are rare and tumor lysis syndrome (TLS) was reported in two cases only $[3,4]$. In one of the cases it was fatal [3]. Here we report another fatal case of TLS following treatment with sorafenib, in a patient

\footnotetext{
Manuscript accepted for publication January 2, 2013

${ }^{a}$ Department of Medicine, Carmel Medical Center, Haifa, and Rheumatology Clinic, Nazareth Hospital, Nazareth, Israel ${ }^{b}$ Department of Pathology, Carmel Medical Center, Haifa, Israel ${ }^{\mathrm{c} C}$ Corresponding author: George Habib, Department of Medicine, Carmel Medical Center Michal 7 str., Haifa 36342, Israel. Email: gshabib@gmail.com
}

doi: http://dx.doi.org/10.4021/jmc1049w with HCC.

\section{Case Report}

A fifty-nine years old male patient who was diagnosed 10 months prior to the current admission with hepatitis B (HB) virus-related $\mathrm{HCC}$ with diffuse infiltration of the liver and evidence of cirrhosis on liver biopsy. There was no evidence of remote metastasis by computerized tomography or bone scan and no clinical evidence of portal hypertension. Prior to that, patient was known to be healthy and carrier of HBs Ag for more than 20 years without special treatment. Alfa fetoprotein (AFP) level at the time of HCC diagnosis was $>1,500 \mathrm{IU} / \mathrm{mL}$ (normal levels $<5 \mathrm{IU} / \mathrm{mL}$ ). Patient had liver transplantation donated by a relative and following the transplantation treated with tacrolimus, mycophenolate mofetil and prednisone. AFP levels following the procedure were normal. 2 months later patient developed bile leakage from the anastomosis of the common bile duct with the gut, resolved by surgery. During his hospital stay patient was diagnosed with pulmonary embolism and treated with low molecular weight heparin, enoxaparin. Patient did well for nearly 7 months when he started to complain of diffuse musculoskeletal pain and weakness. Ultra-sound of the abdomen showed widespread hepatic lesions compatible with metastasis. Computerized tomography (CT) of the abdomen revealed the same finding with enlarged regional lymph nodes. Bone scan showed diffuse bone lesions compatible with metastasis. AFP level was $602 \mathrm{IU} / \mathrm{mL}$. Alkaline phosphatase level was $165 \mathrm{U} / \mathrm{L}$ (normal level up to $124 \mathrm{U} / \mathrm{L}$ ) and gamma glutamyltransaminase (GGT) was $58 \mathrm{U} / \mathrm{L}$ (normal level up to 33). His kidney function and hepatocellular enzyme levels were normal. Liver biopsy was technically unsuccessful and yielded bloody material. Patient was diagnosed with recurrence of HCC. 3 weeks before the current admission, patient was started on sorafenib $400 \mathrm{mg}$ twice a day. At the current admission, patient presented with shortness of breath. Laboratory tests showed acute renal failure with creatinine level of $2.6 \mathrm{mg} / \mathrm{dL}$, urea $105 \mathrm{mg} / \mathrm{dL}$, LDH 6,687 U/L, GOT 2,435 $\mathrm{U} / \mathrm{L}$, GPT $235 \mathrm{U} / \mathrm{L}$, total bilirubin $3 \mathrm{mg} / \mathrm{dL}$, uric acid $11 \mathrm{mg} /$ $\mathrm{dL}$, phosphor $8 \mathrm{mg} / \mathrm{dL}$, white cell count 10,020 cell $/ \mathrm{mL}$, he- 
moglobin $9.2 \mathrm{~g} / \mathrm{dL}, \mathrm{pH}$ (of the blood) 7.12, bicarbonate 10 $\mathrm{mmol} / \mathrm{L}, \mathrm{PCO} 235 \mathrm{mmHg}$, PO2 $120 \mathrm{mmHg}$, O2 saturation $99 \%$ and AFP level was $5.4 \mathrm{IU} / \mathrm{mL}$. Chest $\mathrm{x}$-ray showed mild bilateral pleural effusion. CT showed large areas of necrosis at the liver. Patient was diagnosed with TLS, and started on fluids, bicarbonate and antibiotics. Patient situation deteriorated and was mechanically ventilated. On the fourth day, patient died due to multi-organ failure with levels of LDH reaching 28,000 $\mathrm{U} / \mathrm{L}$ and uric acid $17 \mathrm{mg} / \mathrm{dL}$.

\section{Discussion}

TLS is a very serious complication that results from massive cell necrosis following usually chemotherapy. It is more common in hematologic than solid tumors. In patients with such malignancies and before the initiation chemotherapy, preventive measures including hydration, alkalinization and allopurinol treatment are initiated.

Sorafenib seems to have tremendous effect on tumor cells in some patients with $\operatorname{HCC}(3,4$, and our case). Such effect results in a massive destruction of these cells leading to TLS. TLS in such patients developed 10 to 30 days following sorafenib therapy. In all of them it was acute.

Death occurred in 2 patients (including our patient) at the fourth and thirteenth day after admission. None of the patients received preventive therapy for the development of
TLS and there are no special recommendations according to the manufacturer instructions.

Although the number of reported cases of TLS following sorafenib treatments for advanced HCC is very small, we suggest using preventing measures prior to starting such therapy. We recommend either starting with a low dose and raising it slowly while the patient is well hydrated, or starting with a full dose, only after the patient had been already on allopurinol, under alkalinization and well hydrated.

\section{References}

1. Gauthier A, Ho M. Role of sorafenib in the treatment of advanced hepatocellular carcinoma: An update. Hepatol Res. 2013;43(2):147-154.

2. Llovet JM, Ricci S, Mazzaferro V, Hilgard P, Gane E, Blanc JF, de Oliveira AC, et al. Sorafenib in advanced hepatocellular carcinoma. N Engl J Med. 2008;359(4):378390.

3. Huang WS, Yang CH. Sorafenib induced tumor lysis syndrome in an advanced hepatocellular carcinoma patient. World J Gastroenterol. 2009;15(35):4464-4466.

4. Shiozawa K, Watanabe M, Takenaka H, Nagai H, Ishii K, Sakai K, Sumino Y. Tumor lysis syndrome after sorafenib for hepatocellular carcinoma: a case report. Hepatogastroenterology. 2010;57(101):688-690. 